Sensitivity studies for third-generation gravitational wave observatories

This article has been downloaded from IOPscience. Please scroll down to see the full text article.

2011 Class. Quantum Grav. 28094013

(http://iopscience.iop.org/0264-9381/28/9/094013)

View the table of contents for this issue, or go to the journal homepage for more

Download details:

IP Address: 194.94.224.254

The article was downloaded on 16/01/2012 at 13:16

Please note that terms and conditions apply. 


\title{
Sensitivity studies for third-generation gravitational wave observatories
}

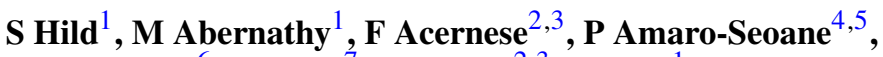 \\ N Andersson $^{6}, \mathbf{K}_{\text {Arun }}{ }^{7}, \mathbf{F}$ Barone $^{2,3}$, B Barr $^{1}$, M Barsuglia $^{8}$, M Beker $^{9}$, \\ N Beveridge ${ }^{1}, \mathbf{S}$ Birindelli ${ }^{10}$, S Bose $^{11}, \mathbf{L ~ B o s i ~}^{12}$, S Braccini $^{13}$, \\ C Bradaschia $^{13}$, T Bulik ${ }^{14}$, E Calloni $^{2,15}$, G Cella $^{13}$, \\ E Chassande Mottin ${ }^{8}$, S Chelkowski ${ }^{16}$, A Chincarini ${ }^{17}$, J Clark ${ }^{18}$, \\ E Coccia ${ }^{19,20}$, C Colacino ${ }^{13}$, J Colas ${ }^{21}$, A Cumming ${ }^{1}$, L Cunningham ${ }^{1}$,

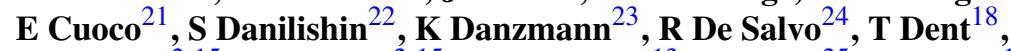 \\ R De Rosa ${ }^{2,15}$, L Di Fiore ${ }^{2,15}$, A Di Virgilio ${ }^{13}$, M Doets ${ }^{25}$, V Fafone ${ }^{19,20}$,

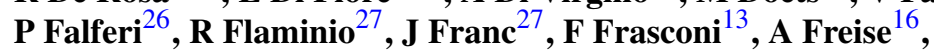 \\ D Friedrich ${ }^{23}$, P Fulda ${ }^{16}$, J Gair ${ }^{28}$, G Gemme ${ }^{17}$, E Genin ${ }^{21}$, A Gennai ${ }^{16}$, \\ A Giazotto ${ }^{13,21}$, K Glampedakis ${ }^{29}, \mathbf{C ~ G r a ̈ f}^{23}, \mathbf{M ~ G r a n a t a}^{8}$, H Grote $^{23}$,

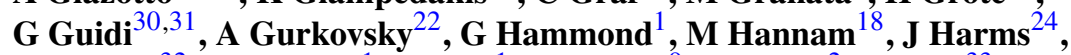

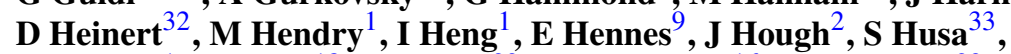 \\ S Huttner ${ }^{1}$, G Jones ${ }^{18}$, F Khalili ${ }^{22}$, K Kokeyama ${ }^{16}$, K Kokkotas ${ }^{29}$, \\ B Krishnan ${ }^{4}$, $\mathbf{T ~ G ~ F ~ L i ~}{ }^{9}, \mathbf{M ~ L o r e n z i n i ~}^{30}, \mathbf{H ~ L u ̈ c k}^{23}$, E Majorana ${ }^{34}$,

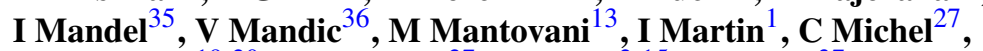

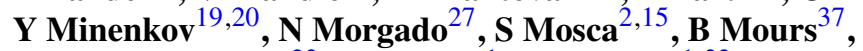 \\ H Müller-Ebhardt ${ }^{23}$, P Murray $^{1}$, R Nawrodt ${ }^{1,32}$, J Nelson $^{1}$, \\ R Oshaughnessy ${ }^{38}, \mathbf{C}$ D Ott $^{39}, \mathbf{C}$ Palomba $^{34}, \mathbf{A ~ P a o l i ~}^{21}, \mathbf{G}$ Parguez $^{21}$, \\ A Pasqualetti ${ }^{21}, \mathbf{R}$ Passaquieti $^{13,40}$, D Passuello ${ }^{13}$, L Pinard $^{27}$, \\ W Plastino ${ }^{41}$, R Poggiani ${ }^{13,40}$, P Popolizio ${ }^{21}$, M Prato ${ }^{17}$, M Punturo ${ }^{12,21}$,

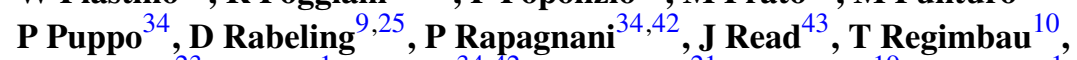

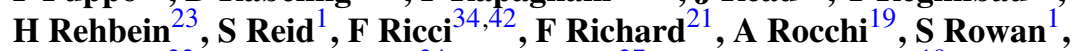 \\ A Rüdiger ${ }^{23}$, L Santamaría ${ }^{24}$, B Sassolas ${ }^{27}$, B Sathyaprakash ${ }^{18}$, \\ R Schnabel $^{23}$, C Schwarz $^{32}$, P Seidel $^{32}$, A Sintes $^{33}$, K Somiya ${ }^{39}$, \\ F Speirits ${ }^{1}$, K Strain ${ }^{1}$, S Strigin ${ }^{22}$, P Sutton $^{18}, \mathbf{S}$ Tarabrin $^{23}$, \\ A Thüring ${ }^{23}, \mathbf{J}$ van den Brand ${ }^{9,25}, \mathbf{M}$ van Veggel ${ }^{1}, \mathbf{C}$ van den Broeck $^{9}$, \\ A Vecchio ${ }^{16}$, J Veitch ${ }^{18}$, F Vetrano ${ }^{30,31}$, A Vicere ${ }^{30,31}, \mathbf{S}$ Vyatchanin $^{22}$, \\ B Willke ${ }^{23}, \mathbf{G ~ W o a n}^{1}$ and K Yamamoto ${ }^{44}$

\footnotetext{
${ }^{1}$ SUPA, School of Physics and Astronomy, The University of Glasgow, Glasgow, G12 8QQ, UK 2 INFN, Sezione di Napoli, Italy

${ }^{3}$ Università di Salerno, Fisciano, I-84084 Salerno, Italy

D-14476 Potsdam, Germany

5 Institut de Ciències de 1'Espai (CSIC-IEEC), Campus UAB, Torre C-5, parells, $2^{\text {na }}$ planta,

ES-08193, Bellaterra, Barcelona, Spain

${ }^{6}$ University of Southampton, Southampton SO17 1BJ, UK

${ }^{7}$ LAL, Université Paris-Sud, IN2P3/CNRS, F-91898 Orsay, France

${ }^{8}$ AstroParticule et Cosmologie (APC), CNRS; Observatoire de Paris, Université Denis Diderot, Paris VII, France

${ }^{9}$ Nikhef, Science Park 105, 1098 XG Amsterdam, The Netherlands
} \\ ${ }^{4}$ Max Planck Institute for Gravitational Physics (Albert Einstein Institute) Am Mühlenberg 1,
}


${ }^{10}$ Université Nice 'Sophia-Antipolis', CNRS, Observatoire de la Côte d'Azur, F-06304 Nice, France

${ }^{11}$ Washington State University, Pullman, WA 99164, USA

12 INFN, Sezione di Perugia, I-6123 Perugia, Italy

13 INFN, Sezione di Pisa, Italy

14 Astronomical Observatory, University of warsaw, Al Ujazdowskie 4, 00-478 Warsaw, Poland

15 Università di Napoli 'Federico II', Complesso Universitario di Monte S. Angelo, I-80126

Napoli, Italy

16 University of Birmingham, Birmingham, B15 2TT, UK

17 INFN, Sezione di Genova, I-16146 Genova, Italy

18 Cardiff University, Cardiff, CF24 3AA, UK

19 INFN, Sezione di Roma Tor Vergata I-00133 Roma, Italy

${ }^{20}$ Università di Roma Tor Vergata, I-00133, Roma, Italy

21 European Gravitational Observatory (EGO), I-56021 Cascina (Pi), Italy

22 Moscow State University, Moscow, 119992, Russia

23 Max-Planck-Institut für Gravitationsphysik and Leibniz Universität Hannover, D-30167 Hannover, Germany

${ }^{24}$ LIGO, California Institute of Technology, Pasadena, CA 91125, USA

25 VU University Amsterdam, De Boelelaan 1081, 1081 HV, Amsterdam, The Netherlands

${ }^{26}$ INFN, Gruppo Collegato di Trento, Sezione di Padova; Istituto di Fotonica e Nanotecnologie, CNR-Fondazione Bruno Kessler, I-38123 Povo, Trento, Italy

${ }^{27}$ Laboratoire des Matériaux Avancés (LMA), IN2P3/CNRS, F-69622 Villeurbanne, Lyon, France

${ }^{28}$ University of Cambridge, Madingley Road, Cambridge, CB3 OHA, UK

29 Theoretical Astrophysics (TAT) Eberhard-Karls-Universität Tübingen, Auf der Morgenstelle 10, D-72076 Tübingen, Germany

${ }^{30}$ INFN, Sezione di Firenze, I-50019 Sesto Fiorentino, Italy

${ }^{31}$ Università degli Studi di Urbino 'Carlo Boapos;, I-61029 Urbino, Italy

32 Friedrich-Schiller-Universität Jena PF, D-07737 Jena, Germany

${ }^{33}$ Departament de Fisica, Universitat de les Illes Balears, Cra. Valldemossa Km. 7.5, E-07122

Palma de Mallorca, Spain

${ }^{34}$ INFN, Sezione di Roma 1, I-00185 Roma, Italy

35 Department of Physics and Astronomy, Northwestern University, Evanston, IL 60208, USA

36 University of Minnesota, Minneapolis, MN 55455, USA

${ }^{37}$ LAPP-IN2P3/CNRS, Université de Savoie, F-74941 Annecy-le-Vieux, France

38 The Pennsylvania State University, University Park, PA 16802, USA

${ }^{39}$ Caltech-CaRT, Pasadena, CA 91125, USA

${ }^{40}$ Università di Pisa, I-56127 Pisa, Italy

${ }^{41}$ INFN, Sezione di Roma Tre and Università di Roma Tre, Dipartimento di Fisica, I-00146

Roma, Italy

42 Università 'La Sapienza', I-00185 Roma, Italy

43 University of Mississippi, MS38677, USA

${ }^{4}$ INFN, sezione di Padova, via Marzolo 8, 35131 Padova, Italy

E-mail: stefan.hild@glasgow.ac.uk

Received 4 December 2010, in final form 7 January 2011

Published 18 April 2011

Online at stacks.iop.org/CQG/28/094013

\section{Abstract}

Advanced gravitational wave detectors, currently under construction, are expected to directly observe gravitational wave signals of astrophysical origin. The Einstein Telescope (ET), a third-generation gravitational wave detector, has been proposed in order to fully open up the emerging field of gravitational wave astronomy. In this paper we describe sensitivity models for ET and investigate potential limits imposed by fundamental noise sources. A special focus is set on evaluating the frequency band below $10 \mathrm{~Hz}$ where a complex 
mixture of seismic, gravity gradient, suspension thermal and radiation pressure noise dominates. We develop the most accurate sensitivity model, referred to as ET-D, for a third-generation detector so far, including the most relevant fundamental noise contributions.

PACS numbers: $04.80 . \mathrm{Nn}, 95.75 . \mathrm{Kk}$

(Some figures in this article are in colour only in the electronic version)

\section{Introduction}

The currently operating gravitational wave (GW) detectors LIGO [1], Virgo [2], GEO 600 [3] and TAMA [4] are based on extremely sensitive Michelson interferometers. While the sensitivity achieved by these first-generation detectors is mainly limited by shot noise, mirror thermal noise and seismic noise, for the second generation of instruments, such as Advanced LIGO [5], Advanced Virgo [6], GEO-HF [7] and LCGT [8], additional fundamental noise sources will start to play a role toward the low-frequency end of the detection band: thermal noise of the test mass suspension, photon radiation pressure noise and seismically driven gravity gradients acting on the test masses. These three sources of noise will become even more important for third-generation GW observatories such as the Einstein Telescope (ET) $[9,10]$, as these detectors aim to significantly increase the detection band toward frequencies as low as a few $\mathrm{Hz}[11,13]$. Therefore, major parts of the ET design are driven by exactly these noise sources. An overview of the importance of the sub-10 Hz band for astrophysical and cosmological analyses can be found in [9].

In this paper we will give an overview of the currently ongoing ET design activities with a special focus on the modelling of the achievable sensitivity taking the most important fundamental noise sources into account. The first sensitivity estimate for a third-generation interferometer was described in $[11,12]$ and was based on a single interferometer covering the full frequency range from about $1 \mathrm{~Hz}$ to $10 \mathrm{kHz}$. In the following we will refer to this sensitivity curve as ET-B. Subsequently we developed a more realistic design, taking cross-compatibility aspects of the various involved technologies into account. This led to the so-called xylophone design, in which one GW detector is composed of two individual interferometers: A low-power cryogenic low-frequency interferometer and a high-power room temperature high-frequency interferometer. A detailed description of this xylophone detector sensitivity, in the following referred to as ET-C, can be found in [13]. The ET-C configuration will serve as a starting point for the investigations described in this paper. We improved the sensitivity models for ET by including additional new noise sources as well as by amending and updating noise contributions already previously included. These improvements, which led to a new sensitivity estimate, referred to as ET-D, will be presented and discussed in this paper.

In section 2 we discuss seismic and gravity-gradient noise, followed by the quantum-noise contribution in section 3. Thermal noise of the suspensions and test masses will be presented in section 4. An improved noise budget for ET is then given in section 5. We conclude with a brief overview of the configuration of a full third-generation observatory, consisting of several GW detectors.

\section{Seismic isolation and gravity-gradient noise}

Seismic noise couples into the differential arm length of a GW detector via two main paths. First of all, seismic excitation can mechanically couple through the suspension and seismic 

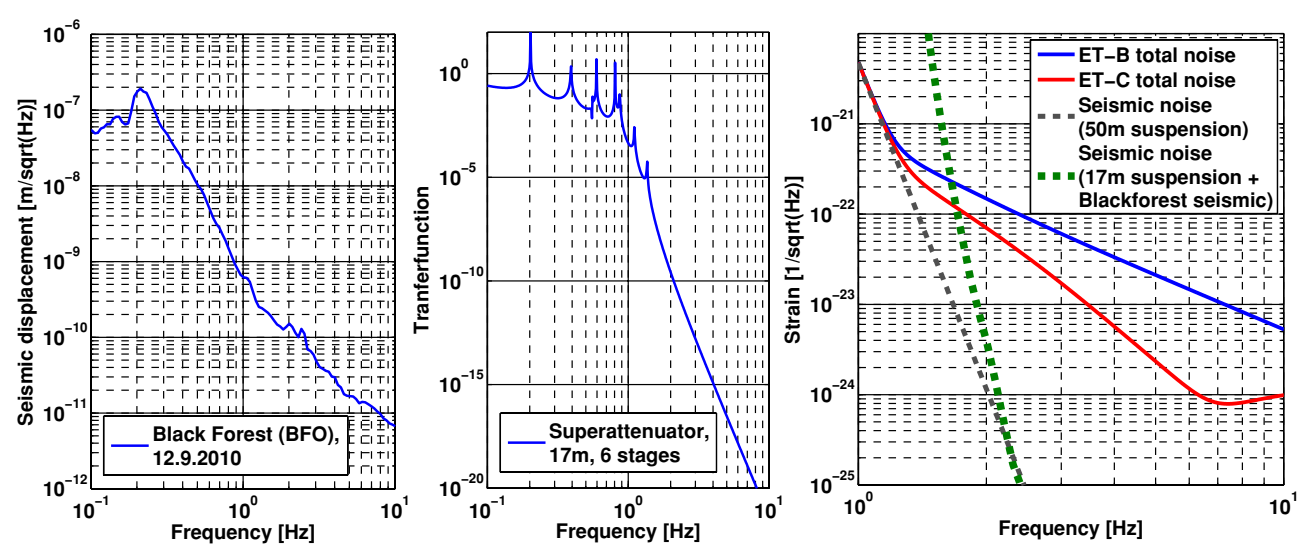

Figure 1. Seismic noise spectrum from an underground location in the Black Forest, Germany (left-hand panel). Transfer function of a superattenuator consisting of six stages with an overall height of $17 \mathrm{~m}$ (center panel). The right-hand panel shows the resulting seismic noise contribution for the $17 \mathrm{~m}$ superattenuator for the seismic excitation at the Black Forest site (green dashed line). For comparison ET-B and ET-C are also plotted. Their seismic noise contribution is based on the assumption of a generic five-stage $50 \mathrm{~m}$ suspension.

isolation systems. Second, seismic noise excites density fluctuations in the environment of the GW detector, which couple via gravitational attraction to the test-mass position. In the following we will refer to these two noise sources as seismic noise and gravity-gradient noise, respectively. The main difference between these two noise sources is that while seismic noise can be reduced by application of complex seismic isolation systems, the only guaranteed way to reduce the gravity-gradient noise is to reduce the initial seismic excitation. ${ }^{45}$ Therefore, third-generation GW detectors are proposed to be built in quiet underground locations.

The seismic noise contribution of the low-frequency interferometer of ET-C was based on a seismic excitation of $5 \times 10^{-9} \mathrm{~m} / \sqrt{\mathrm{Hz}} / f^{2}$ (where $f$ is the frequency in $\mathrm{Hz}$ ) and a generic $50 \mathrm{~m}$ tall seismic isolation system consisting of five passive pendulum stages, each of $10 \mathrm{~m}$ height. A more realistic seismic isolation design, based on the Virgo superattenuator concept $[14,15]$, has been developed recently [16]. To achieve a lower cut-off frequency the height of the individual pendulum stages of the superattenuator will be extended to $2 \mathrm{~m}$ per stage. The overall isolation of the proposed modified superattenuator, consisting of six pendulum stages (each stage providing horizontal as well as vertical isolation) and a total height of $17 \mathrm{~m}$, is shown in the center panel of figure 1. Using the seismic excitation level, measured in an underground facility of the Black Forest Observatory (BFO) [17, 18], shown in the left panel of figure 1, we can derive the expected seismic noise contribution to the ET noise budget. The result is shown in the right-hand panel of figure 1. Reducing the height of the seismic isolation system from 50 to $17 \mathrm{~m}$ increases the cut-off frequency only slightly from about 1.2 to $1.7 \mathrm{~Hz}$.

Gravity-gradient noise has been described in detail [20-22]. In our simulations we estimate the power spectral density of the gravity-gradient noise contribution as

$$
N_{\mathrm{GG}}(f)^{2}=\frac{4 \cdot \beta^{2} \cdot G^{2} \cdot \rho_{r}^{2}}{L^{2} \cdot f^{4}} \cdot X_{\mathrm{seis}}^{2},
$$

\footnotetext{
45 Many promising gravity-gradient noise subtraction schemes have been suggested in the literature [19]. However, as none of these schemes has been demonstrated so far, we do not consider them in this paper.
} 

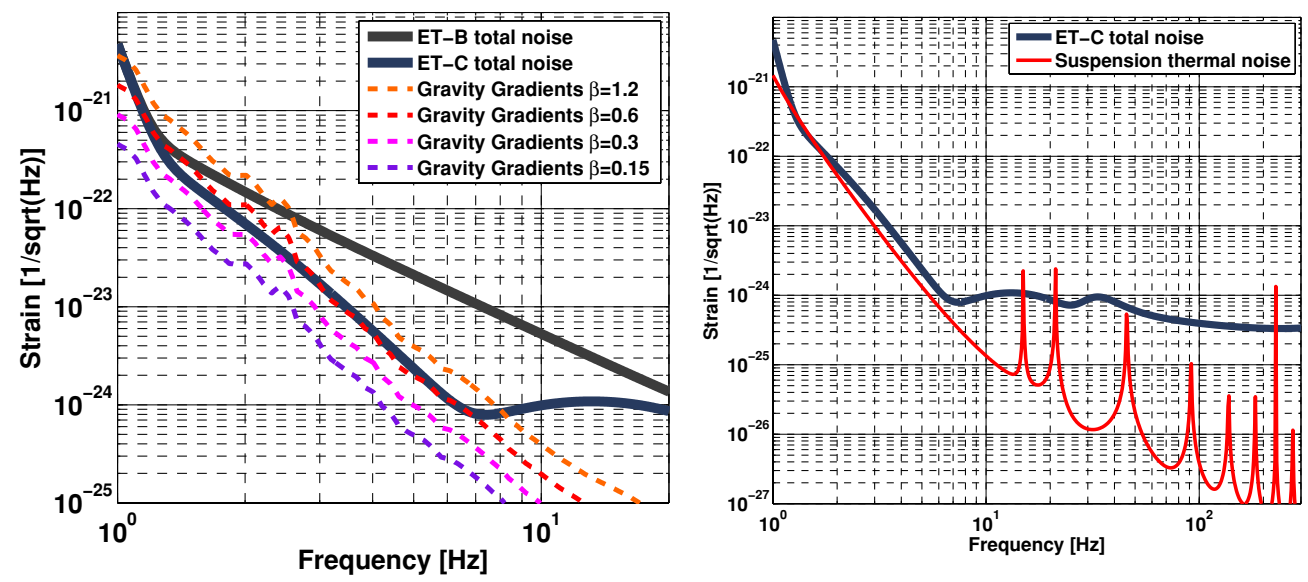

Figure 2. Left panel: gravity-gradient noise contribution to $\mathrm{ET}$, for various $\beta$ values, assuming the BFO spectrum shown in figure 1 as the seismic excitation level. Right panel: suspension thermal noise of the low-frequency interferometer of ET as described in [34].

where $G$ is the gravitational constant, $\rho_{r}$ is the density of the rock around the GW detector, $L$ is the arm length of the interferometer, $f$ is the frequency and $X_{\text {seis }}^{2}$ is the power spectral density of the ground motion. $\beta$ accounts for the actual coupling transfer function from seismic excitation to the differential arm length noise and depends for instance on the wave type of the seismic excitation (e.g. ratio of $\mathrm{P}$ and $\mathrm{S}$ waves) and soil characteristics.

Within the ET design study we carried out a campaign of measuring the seismic noise in various underground locations across Europe. These measurements have indicated that a couple of the test locations show a seismic excitation level similar to or below the BFO measurement [23]. Therefore, we assumed the BFO seismic excitation as a conservative estimate of a potential ET site. The left panel of figure 2 shows the corresponding gravitygradient noise contribution at the BFO site for different $\beta$. Since the detailed evaluation of a realistic $\beta$ for the potential ET sites is an ongoing activity, we will use $\beta=0.58$, as given in the literature [20, 21], in the following for the ET-D sensitivity. Please note that our models do not take atmospheric Newtonian noise into account.

\section{Shaping of quantum noise}

Quantum noise, composed of photon shot noise at high frequencies and photon radiation pressure noise at low frequencies, contributes significantly to the overall sensitivity of ET's high-frequency and low-frequency detectors. The high-frequency interferometers will feature a light power stored in the arm cavities of about $3 \mathrm{MW}$ to reduce shot noise, while the low-frequency interferometers make use of only $18 \mathrm{~kW}$ of light power in the arms, in order to reduce the radiation pressure noise. For ET-C the quantum-noise contribution was optimized by making use of detuned (low-frequency interferometer) and tuned (high-frequency interferometer) signal recycling [24, 25], together with an assumed generic quantum-noise reduction of $10 \mathrm{~dB}$ at all frequencies.

Such a broadband quantum-noise reduction can, in principle, be achieved by injecting squeezed light states with a frequency-dependent squeezing angle [26] into the output port of the interferometer. Starting from a frequency-independent squeezing angle, it is possible 

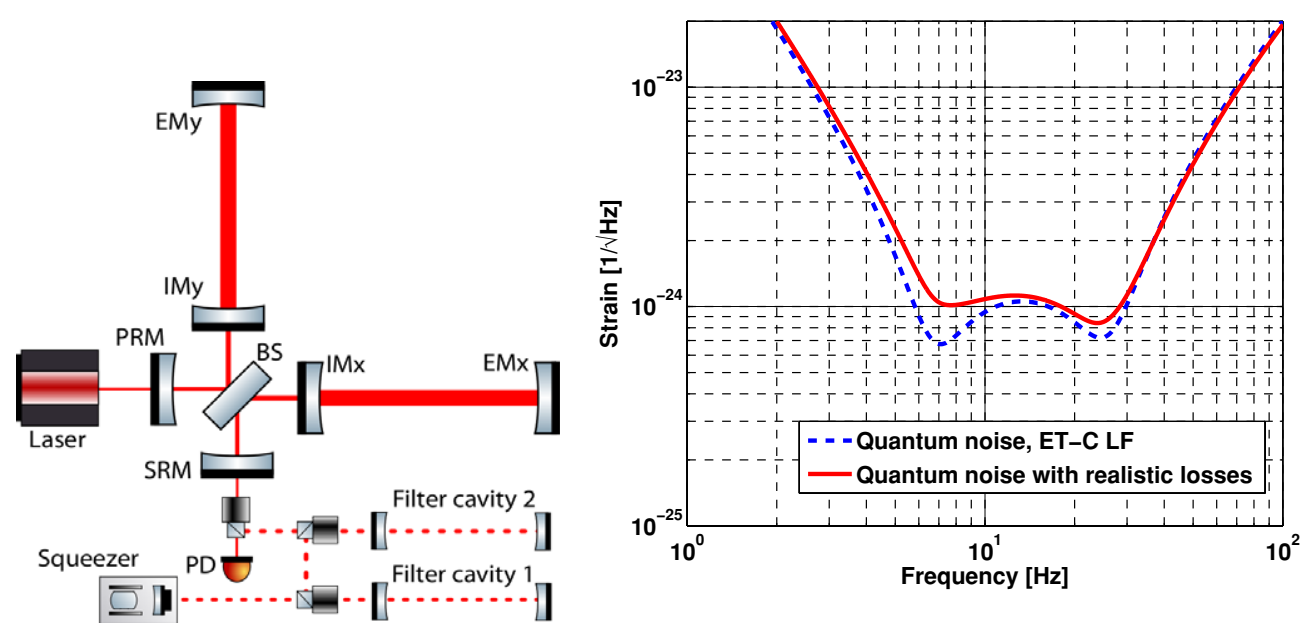

Figure 3. Left panel: simplified schematic of an ET interferometer. Quantum-noise suppression is achieved by the injection of squeezed light states with a frequency-dependent squeezing angle. The frequency-dependent rotation of the squeezing angle can be realized by using the dispersion of filter cavities, on which the squeezed light is reflected. Each ET low-frequency interferometer will require two filter cavities, while each high-frequency interferometer only requires a single filter cavity. Right-hand panel: quantum-noise contribution of the ET low-frequency interferometer, as described in [13] (dashed line) and with squeezing losses from filter cavities taken into account (solid line) [27].

to use the dispersion occurring in reflection of a cavity, to create squeezed light states with a frequency-dependent squeezing angle. Figure 3 shows a simplified schematic of a dual recycled interferometer with arm cavities, consisting of a power recycling mirror (PRM), the beam splitter (BS), the arm cavity mirrors (IM and EM) and the signal recycling mirror (SRM). In addition the injection of the frequency-dependent squeezed light states is also shown; the squeezed light states leave the squeezing source (squeezer) and are reflected at two filter cavities before they are injected via a Faraday rotator into the interferometer mode. Finally, the interferometer output mode, consisting of the squeezed field and the signal field, is detected on the main photo diode (PD).

In general, for an interferometer with signal recycling two filter cavities are necessary: one for compensating the dispersion of the signal recycling resonance and the other to reduce radiation pressure noise. The bandwidth and detuning of the filter cavities depend on the actual optical parameters of the main interferometers (e.g. arm length, SRM reflectivity, tuning of the signal recycling cavity) and need to be matched very accurately to establish the full sensitivity improvement of the squeezed-light injection. For the ET-D high-frequency interferometer we have the special case that only one filter cavity will be required, as it employs tuned signal recycling with a bandwidth significantly larger than the crossover frequency of radiation pressure and shot noise.

The major loss mechanism for the squeezed light reflected off the filter cavities originates from the fact that for frequencies close to the resonance of the filter cavities, the squeezed states partly enter the filter cavity and experience unavoidable round-trip losses. We recently performed a detailed analysis of the requirements for the ET filter cavities as well as quantifying the squeezing losses inside the filter cavities [27, 28]. We assumed a squeezing level of $10 \mathrm{~dB}$, an antisqueezing level of $15 \mathrm{~dB}$ and a loss of $75 \mathrm{ppm}$ per round trip inside a filter cavity. 

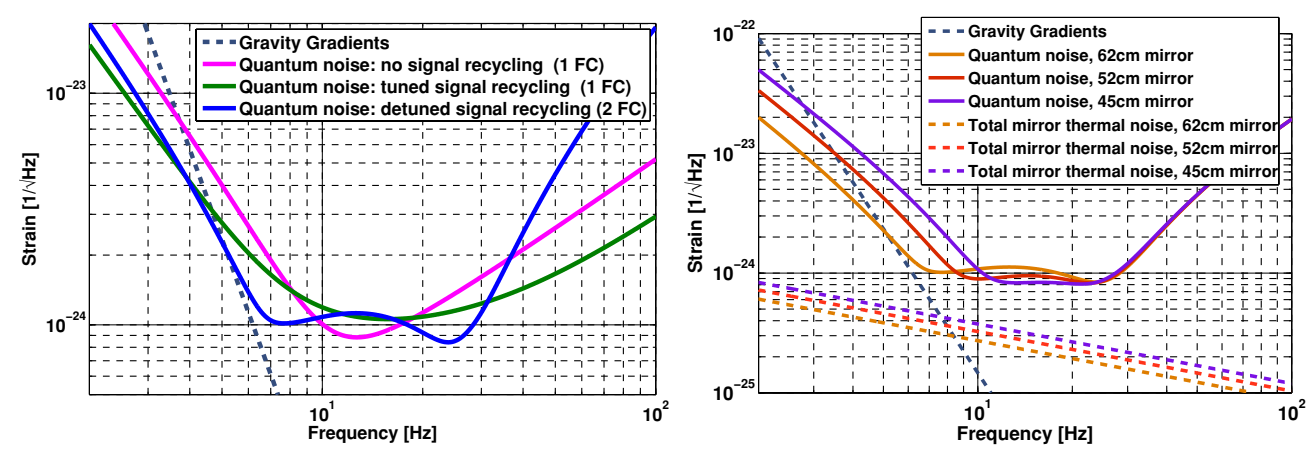

Figure 4. Left: quantum-noise contribution for a low-frequency ET interferometer with different signal recycling options. For ET-D we assumed detuned signal recycling with SRM reflectivity of $80 \%$. Also plotted are the tuned signal recycling configuration using a $30 \%$ reflectivity SRM and quantum noise without any signal recycling. In brackets the number of required filter cavities is stated. Right: quantum-noise and mirror-thermal-noise contributions for different mirror diameters. The aspect ratio is kept constant for all scenarios. Reducing the mirror size (and thus their weight) only slightly increases the mirror thermal-noise contributions, but significantly decreases the sensitivity at low frequencies, due to increased radiation pressure noise.

In order to reduce the influence of the intra-cavity losses, we chose a rather long filter cavity length of $10 \mathrm{~km}$, which allows us to keep the filter cavity finesse at a moderate level. The right-hand plot in figure 3 shows the corresponding quantum-noise contributions of ET-D for the low-frequency interferometer with filter cavity losses included (solid lines).

For the low-frequency interferometer it is also interesting to compare different signal recycling options, properly accounting for the squeezing losses inside the filter cavities. The left-hand plot of figure 4 shows the ET-D configuration, employing detuned signal recycling together with two filter cavities, in comparison to tuned signal recycling as well as an configuration without signal recycling. Both of these latter configurations require only a single filter cavity, but this comes at the price of significantly lower sensitivity in the frequency band of interest $(4-30 \mathrm{~Hz})$.

\section{Thermal-noise contributions}

Brownian fluctuations couple into the differential arm length signal as thermal noise of the test mass itself and of its suspension. Both of these noise contributions can be significantly reduced by lowering the temperature of the test masses and the corresponding suspensions. The Japanese CLIO project [29] has successfully demonstrated the operation of a laser interferometer at cryogenic temperatures. The recently funded LCGT detector [8] is expected to transfer cryogenic technologies to a full-scale second-generation GW detector.

While for the ET high-frequency interferometers even at room temperature the various thermal-noise contributions either do not play a significant role or can be sufficiently reduced by increasing the beam size on the test masses and the use of higher order Laguerre Gauss beam shapes [30] or so-called Mesa beams [31], the ET low-frequency interferometers are expected to operate at cryogenic temperature.

In the frequency band from 1 to $10 \mathrm{~Hz}$ suspension thermal noise is the dominating thermal-noise contribution. When operating the low-frequency interferometer at cryogenic temperatures the last stage suspension does not only need to be compliant with the thermalnoise requirements, but the actual design is also driven by the requirement to extract any 
heat (deposited by the laser beams in the test masses) via the suspension. Our model [34] assumes a mirror temperature of $10 \mathrm{~K}$, silicon fibers of $2 \mathrm{~m}$ length and $3 \mathrm{~mm}$ diameter as well as a temperature of the penultimate mass of $2 \mathrm{~K}$. The right-hand plot of figure 2 shows the simulated suspension thermal-noise contribution for the ET low-frequency detector [34] using a branched system of multiple oscillators consisting of the main mirror, the penultimate mass and the reaction mass $[32,33]$.

Fused silica, which is the material of choice for the test masses of all first-generation GW detectors, has a high dissipation at low temperatures and therefore cannot be used as substrate material for cryogenic test masses [38, 39]. Sapphire and silicon have been proposed as alternative materials $[8,41]$ and there are strong $\mathrm{R}+\mathrm{D}$ efforts to evaluate the optical and mechanical properties of these two candidate materials. In the following we assume silicon as test material for ET, but sapphire would yield similar results. A detailed noise analysis of a cryogenic test mass for ET is given in [40]. The total thermal noise of the test masses is a combination of coating Brownian, substrate Brownian, substrate thermoeleastic and thermooptic noise. Of these four contributions coating Brownian noise is the most important one because in the frequency range of interest it is a factor of about 5 larger than any of the other thermal noises. The power spectral density of coating Brownian noise can be described as

$$
S_{x}(f)=\frac{4 k_{\mathrm{B}} T}{\pi^{2} f Y} \frac{d}{r_{0}^{2}}\left(\frac{Y^{\prime}}{Y} \phi_{\|}+\frac{Y}{Y^{\prime}} \phi_{\perp}\right)
$$

where $f$ is the frequency, $d$ is the total thickness of the coating, $r_{0}$ describes the beam radius, $Y$ and $Y^{\prime}$ are Young's modulus values for the substrate and coating, respectively. $\phi_{\|}$and $\phi_{\perp}$ are the mechanical loss values for the coating for strains parallel and perpendicular to the coating surface [35].

Using silicon test masses ( $62 \mathrm{~cm}$ diameter, $30 \mathrm{~cm}$ thickness) of $10 \mathrm{~K}$, a Young's modulus of silicon of $164 \mathrm{GPa}$, loss angles of $5 \times 10^{-5}$ and $2 \times 10^{-4}$ for the low- and high-refraction coating materials ${ }^{46}$, respectively, and a laser beam radius of $12 \mathrm{~cm}$, we get a total mirror thermal-noise contribution for the low-frequency detector as indicated by the orange dashed line in the right-hand plot of figure 4. In this scenario the mirror thermal noise would be at least a factor of 3 below the quantum noise for all frequencies. Hence, we could in principle consider reducing the beam size on the test masses, which could allow for the reduction of the mirror size. Smaller test masses and smaller laser beams would be advantageous for many aspects of the observatory design, such as for instance the total mass of the cryogenic payload or the mode matching into the arm cavities. However, on the other hand reducing the mirror mass will increase the radiation pressure noise contribution.

The right-hand plot of figure 4 shows a trade-off analysis of the beam size and mirror mass. Starting from a mirror of $62 \mathrm{~cm}$ diameter and $30 \mathrm{~cm}$ thickness which corresponds to a beam radius of $12 \mathrm{~cm}$, we reduce the mirror diameter to $52 \mathrm{~cm}$ and $45 \mathrm{~cm}$, while keeping the aspect ratio of the mirror substrate constant. Using this assumption, already a small reduction in the beam size will increase the radiation pressure noise dramatically and subsequently spoil the sensitivity in the sub-10 Hz band. Therefore, we assume for the low-frequency interferometer of ET-D a reduced beam radius of $9 \mathrm{~cm}$, corresponding to an effective test mass diameter of $45 \mathrm{~cm}$, but at the same time keep the overall test mass weight at about $200 \mathrm{~kg}$.

\footnotetext{
${ }^{46}$ Unfortunately the available measurements indicate higher loss angles for the coating materials at cryogenic temperatures than at room temperature [42]. However, since research on cryogenic coatings has just started, we optimistically assumed that by the time construction of third-generation instruments starts, coatings will be available featuring the same loss angles as current coatings at room temperature [36, 37].
} 
Table 1. Summary of the most important parameters of the ET-D high- and low-frequency interferometers as shown in figure 5. $\mathrm{SA}=$ superattenuator, freq. dep. squeez. = squeezing with frequency-dependent angle.

\begin{tabular}{lll}
\hline Parameter & ET-D-HF & ET-D-LF \\
\hline Arm length & $10 \mathrm{~km}$ & $10 \mathrm{~km}$ \\
Input power (after IMC) & $500 \mathrm{~W}$ & $3 \mathrm{~W}$ \\
Arm power & $3 \mathrm{MW}$ & $18 \mathrm{~kW}$ \\
Temperature & $290 \mathrm{~K}$ & $10 \mathrm{~K}$ \\
Mirror material & Fused silica & Silicon \\
Mirror diameter/thickness & $62 \mathrm{~cm} / 30 \mathrm{~cm}$ & min $45 \mathrm{~cm} / \mathrm{TBD}$ \\
Mirror masses & $200 \mathrm{~kg}$ & $211 \mathrm{~kg}$ \\
Laser wavelength & $1064 \mathrm{~nm}$ & $1550 \mathrm{~nm}$ \\
SR-phase & tuned $(0.0)$ & detuned $(0.6)$ \\
SR transmittance & $10 \%$ & $20 \%$ \\
Quantum-noise suppression & freq. dep. squeez. & freq. dep. squeez. \\
Filter cavities & $1 \times 10 \mathrm{~km}$ & $2 \times 10 \mathrm{~km}$ \\
Squeezing level & $10 \mathrm{~dB}(\mathrm{effective})$ & $10 \mathrm{~dB}(\mathrm{effective})$ \\
Beam shape & LG & TEM \\
Beam radius & $7.25 \mathrm{~cm}$ & $9 \mathrm{~cm}$ \\
Scatter loss per surface & $37.5 \mathrm{ppm}$ & $37.5 \mathrm{ppm}$ \\
Partial pressurefor $\mathrm{H}_{2} \mathrm{O}, \mathrm{H}_{2}, \mathrm{~N}_{2}$ & $10^{-8}, 5 \times 10^{-8}, 10^{-9} \mathrm{~Pa}$ & $10^{-8}, 5 \times 10^{-8}, 10^{-9}$ Pa \\
Seismic isolation & $\mathrm{SA}, 8 \mathrm{~m} \mathrm{tall}$ & mod SA, 17 m tall \\
Seismic (for $f>1 \mathrm{~Hz})$ & $5 \times 10^{-10} \mathrm{~m} / f^{2}$ & $5 \times 10^{-10} \mathrm{~m} / f^{2}$ \\
Gravity-gradient subtraction & none & none \\
\hline
\end{tabular}

\section{Overall sensitivity of the Einstein Telescope}

Table 1 shows the most important parameters of the ET-D interferometers. The corresponding noise budgets for the high- and low-frequency interferometers are shown in figure 5. The sensitivity of the low-frequency detector is limited by seismic noise below $1.7 \mathrm{~Hz}$, while gravity-gradient noise directly limits in the frequency band between 1.7 and $6 \mathrm{~Hz}$. For all frequencies above $6 \mathrm{~Hz}$ (apart from the violin mode resonances) quantum noise is the limiting noise source.

The crossover frequency of the sensitivities of the low- and high-frequency interferometers is at about $35 \mathrm{~Hz}$. Above this frequency the high-frequency interferometer is limited only by two noise sources: mirror thermal-noise limits the sensitivity between 40 and $200 \mathrm{~Hz}$, while the high-frequency section is limited by quantum noise.

Figure 6 shows the evolution of the sensitivity models for ET over the past few years. The very first strawman design was based on a single cryogenic interferometer covering the full frequency range of interest (ET-B) [11]. The introduction of the xylophone design resulted in the ET-C sensitivity. In this paper we significantly refined the xylophone concept and obtained the ET-D sensitivity, which is slightly worse than the ET-C sensitivity, but much more realistic. The loss of sensitivity below $1.7 \mathrm{~Hz}$ can be attributed to the application of a more realistic suspension model yielding increased seismic noise. The slightly worse sensitivity in the range between 2 and $8 \mathrm{~Hz}$ is the result of the inclusion of suspension thermal noise for the cryogenic interferometer as well as the omission of any potential gravity-gradient subtraction. The most 

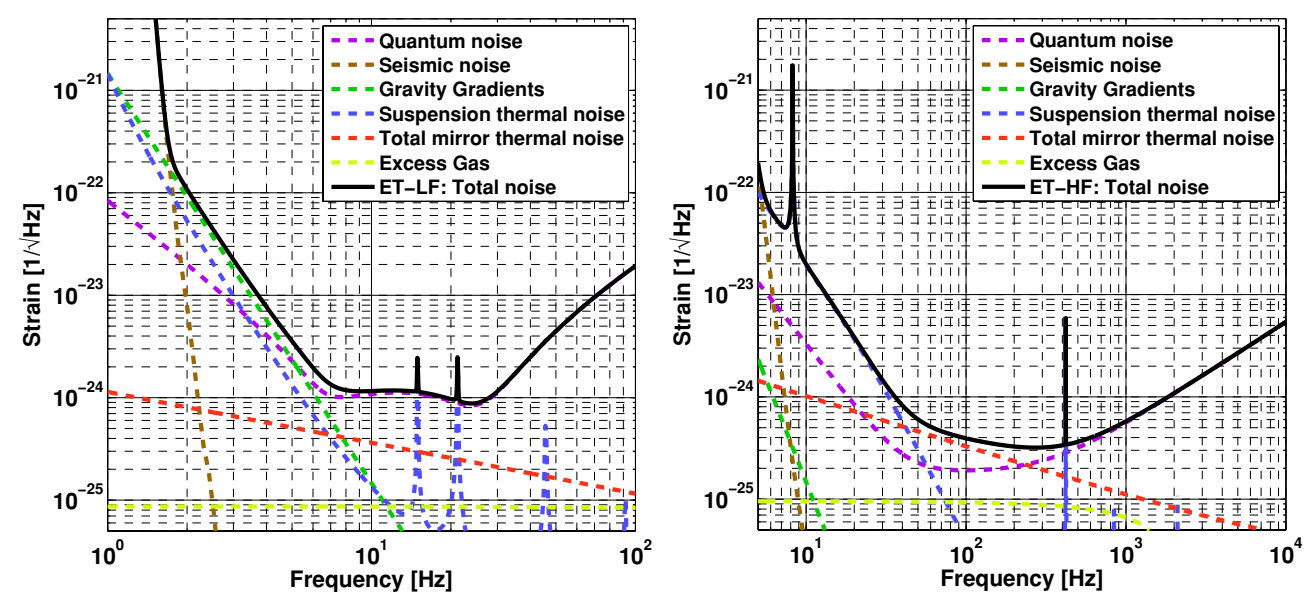

Figure 5. Noise budgets for the ET-D low- and high-frequency interferometers, using the parameters given in table 1.

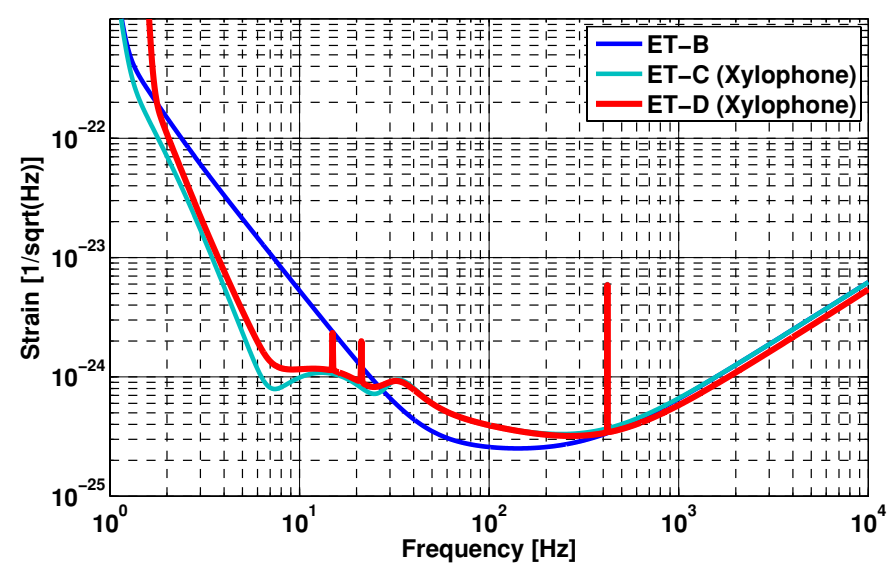

Figure 6. Historical evolution of sensitivity models for the Einstein Telescope, starting from a single cryogenic broadband detector (ET-B) [11], over the initial xylophone design (ET-C) [13] to the ET-D sensitivity described in this paper.

significant difference between ET-C and ET-D shows up between 6 and $10 \mathrm{~Hz}$ and originates from including realistic squeezing losses experienced inside the filter cavities.

\section{Building a full third-generation observatory}

As discussed in the previous sections, one ET detector, covering the full detection band will be made of two individual interferometers, one for low frequencies and one for high frequencies. However, the full ET observatory will consist of three detectors arranged in a triangular shape [43], thus ultimately six interferometers will form the whole observatory.

It needs to be pointed out that the sensitivities described in this paper refer to a single pair of low- and high-frequency interferometers of $10 \mathrm{~km}$ arm length and an opening angle of $90^{\circ}$ (as shown in subplot A of figure 7). The actual effective sensitivity of the full triangular ET 

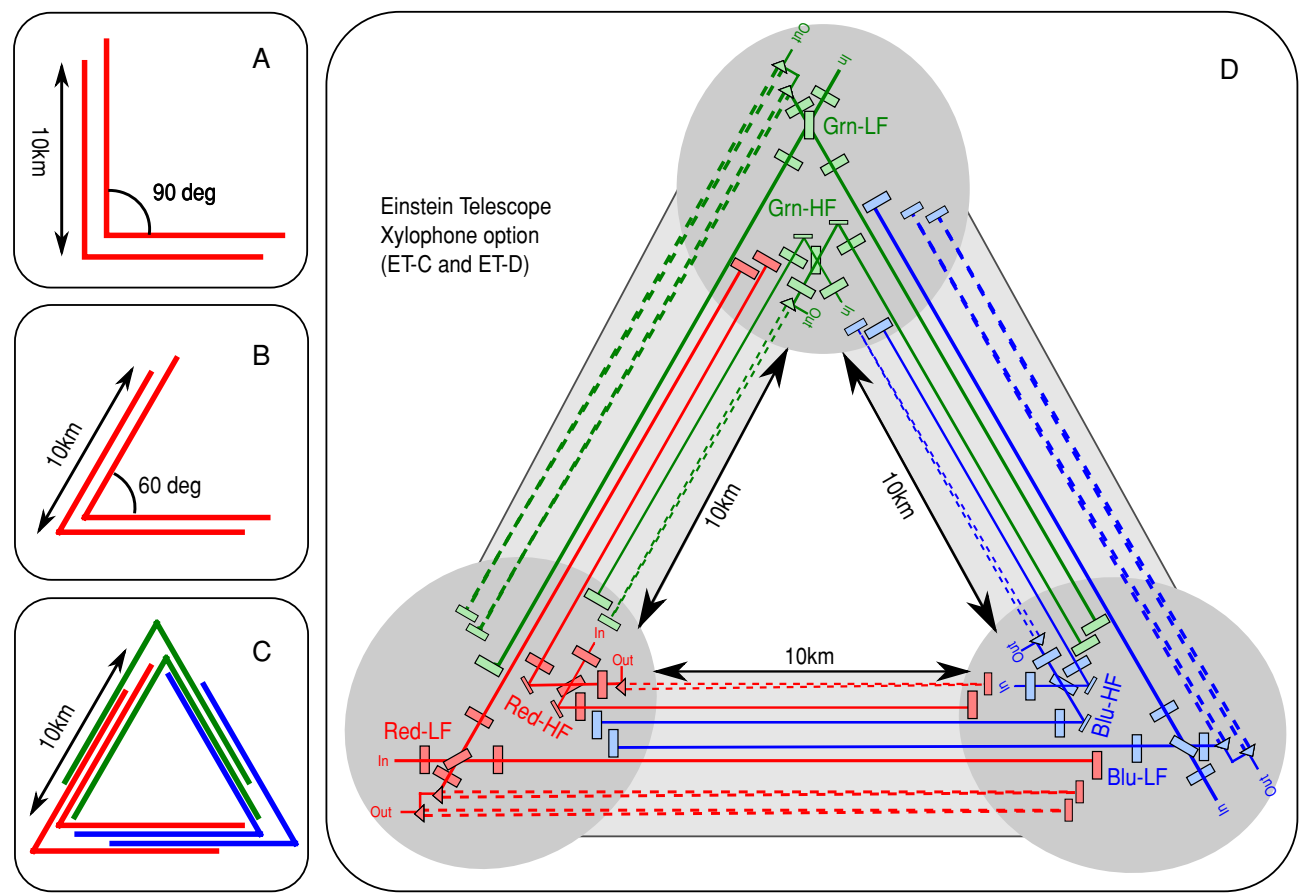

Figure 7. Different interferometer configurations considered in this paper. All sensitivities shown in this paper refer to a pair of low- and high-frequency interferometers forming a single detector of $10 \mathrm{~km}$ arm length and an opening angle of $90^{\circ}$. However, the full ET observatory will consist of three detectors with a $60^{\circ}$ opening angle, arranged in the shape of a triangle. The solid lines represent the main laser beams, while dashed lines indicate squeezed light beams.

observatory depends on the the orientation and polarization of the source of interest. Let us assume a source directly positioned above the observatory emitting $\mathrm{GW}$ of plus polarization. In case of the configuration $\mathrm{A}$ in figure 7 , the sensitivity, $h(f)_{90}$, is then exactly represented by the ET-D trace in figure 6.

If we now decrease the opening angle of the two interferometers to $60^{\circ}$ (see configuration $\mathrm{B}$ in figure 7), the effective sensitivity for plus polarized $\mathrm{GW}$ is given by

$$
h(f)_{60}=\frac{1}{\sin \left(60^{\circ}\right)} \times h(f)_{90}=1.155 \times h(f)_{90},
$$

which is equivalent to shifting the ET-D curve in figure 6 up by about $15 \%$.

Finally, if we consider the full triangle for a plus polarized source, we find that the blue and red detectors in configuration $\mathrm{C}$ of figure 7 have the same sensitivity, while no signal shows up in the green detector. If we combine the red and blue detectors, the noise of the two needs to be added in quadrature because it is uncorrelated, while the coherent signals need to be added up linearly. Thus, the overall sensitivity of the full triangular observatory can be written as

$$
h(f)_{\Delta}=\frac{1}{\sqrt{\left(\sin \left(60^{\circ}\right)\right)^{2}+\left(\sin \left(60^{\circ}\right)\right)^{2}}} \times h(f)_{90}=0.816 \times h(f)_{90},
$$

which would be equivalent to shifting the ET-D curve in figure 6 down by about $18 \%$.

Subplot D of figure 7 shows a schematic drawing of the full ET observatory configuration assumed for the ET-D sensitivity. Included are all main mirrors of the six interferometers, as 
well as a total of nine required filter cavities for the frequency-dependent squeezing. In total this sums up to seven laser beams per tunnel.

\section{Summary and future plans}

In this paper we described a snapshot of the ongoing sensitivity studies for a European thirdgeneration GW observatory. The ET-D sensitivity represents a much more realistic sensitivity compared to previous models, because we included new noise sources as well as improved the accuracy of several already previously included fundamental noise sources. Key points of the new sensitivity model are the inclusion of suspension thermal noise and a realistic seismic isolation system for the low-frequency interferometer and the proper accounting for squeezing losses inside the filter cavities. Finally it needs to be pointed out that the current model does not rely on any subtraction techniques for gravity-gradient noise.

In the future, we plan to further refine our sensitivity models by including noise contributions from optical components outside the arm and filter cavities as well as by taking technical contributions such as laser frequency and laser amplitude noise into account.

\section{Acknowledgments}

The ET-D sensitivity data are available at http://www.et-gw.eu/etsensitivities. The authors are grateful for support from their hosting institutions and national funding agencies. This work has been performed with the support of the European Commission under the Framework Programme 7 (FP7) 'Capacities', project Einstein Telescope (ET) design study (grant agreement 211743) (http://www.et-gw.eu/).

\section{References}

[1] Abbott B P et al 2009 Rep. Prog. Phys. 72076901

[2] Acernese F et al 2008 Class. Quantum Grav. 25114045

[3] Grote H 2008 for the LSC Class. Quantum Grav. 25114043

[4] Takahashi R (the TAMA Collaboration) 2004 Status of TAMA300 Class. Quantum Grav. 21 S403-8

[5] Harry G M (for the LIGO Scientific Collaboration) 2010 Class. Quantum Grav. 27084006

[6] wwwcascina.virgo.infn.it/advirgo/

[7] Willke B et al 2006 Class. Quantum Grav. 23 S207-14

[8] Kuroda K (the LCGT Collaboration) 2006 Class. Quantum Grav. 23 S215-21

[9] Punturo M et al 2010 Class. Quantum Grav. 27084007

[10] http://www.et-gw.eu/

[11] Hild S et al 2008 arXiv:0810.0604v2 [gr-qc]

[12] Freise A et al 2010 General Relativity and Gravitation 108

[13] Hild S et al 2010 Class. Quantum Grav. 27015003

[14] Ballardin G et al 2001 Rev. Sci. Instrum. 72 3643-52

[15] Braccini S et al 2005 Astropart. Phys. 23 557-65

[16] Braccini S presentation at GWADW 2010 (Kyoto), available at: http://gw.icrr.u-tokyo.ac.jp/gwadw2010/ program/2010_GWADW_Braccini.ppt

[17] http://www.gik.uni-karlsruhe.de/en/bfo.html

[18] Forbriger T 2010 Black Forest Observatory (BFO) Schiltach personal communication

[19] Beker M et al 2010 General Relativity and Gravitation (The Netherlands: Springer) pp 1-34

[20] Saulson P R 1984 Phys. Rev. D 30 732-6

[21] Beccaria M et al 1998 Class. Quantum Grav. 15 3339-62

[22] Hughes S A and Thorne K S 1998 Phys. Rev. D 58122002

[23] Beker M presentation at GWADW 2010 (Kyoto) available at: http://gw.icrr.u-tokyo.ac.jp/gwadw2010/ program/2010_GWADW_Beker.pdf

[24] Meers B J 1988 Phys. Rev. D 38 2317-26 
[25] Hild S et al 2007 Class. Quantum Grav. 24 1513-23

[26] Kimble H J et al 2002 Phys. Rev. D 65022002

[27] Thüring A 2010 Filter cavities for GWADS, ET-0104A-10 available at: https://tds.ego-gw.it/itf/tds/index.php

[28] Thüring A et al Filter cavities for advanced gravitational wave detectors and their technical requirements (in preparation)

[29] Miyoki S et al 2010 J. Phys.: Conf. Ser. 203012075

[30] Chelkowski S et al 2009 Phys. Rev. D 79122002

[31] Miller J et al 2008 Class. Quantum Grav. 25235016

[32] Puppo P 2010 J. Phys.: Conf. Ser. 228012031

[33] Puppo P and Ricci F 2010 General Relativity and Gravitation (The Netherlands: Springer) pp 1-13

[34] Ricci F presentation at GWADW 2010 (Kyoto) available at: http://gw.icrr.u-tokyo.ac.jp/gwadw2010/program/ 2010_GWADW_Ricci.pdf

[35] Harry G M et al 2002 Class. Quantum Grav. 19 897-917

[36] Harry G M et al 2006 Appl. Opt. 45 1569-74

[37] Harry G M et al 2007 Class. Quantum Grav. 24 405-15

[38] Anderson O L and Bommel H E 1955 J. Am. Ceram. Soc. 38 125-31

[39] Wiedersich J et al 2000 Phys. Rev. Lett. 84 2718-21

[40] Franc J et al 2009 ET-021-09 available at https://tds.ego-gw.it/itf/tds/index.php

[41] Rowan S et al 2003 Proc. SPIE $\mathbf{4 8 5 6} 292$

[42] Martin I et al 2008 Class. Quantum Grav. 25055005

[43] Freise A et al 2009 Class. Quantum Grav. 26085012 